\title{
A case report of the unilateral approach to the ruptured right ICA aneurysm together with tuberculum sella meningioma and left ICA aneurysm
}

\author{
Jiu Geng Feng ${ }^{1 \dagger}$, Min Jun Wei ${ }^{1 \dagger}$, Rong Hua Feng ${ }^{2}$, Tao Hong ${ }^{1}$ and Chun Hui Zeng ${ }^{1 *}$
}

\begin{abstract}
Background: Tuberculum sellae meningioma associated with aneurysm is extremely rare with a limited number of individual cases previously reported. Because of the complicated anatomical structure of the skull base, comorbid disease in the sellae region is a challenging problem and is associated with high mortality in neurosurgery.

Case presentation: This case report describes a rare case of comorbid bilateral internal carotid artery aneurysms and tuberculum sellae meningioma. This 58-year-old female presented with headache and nausea. She had lost her light perception in the right eye, and the right optic disc was degenerated. She also had a 5-year history of hypertension. This patient underwent a right pterional craniotomy, and we accomplished to clip the bilateral intracranial aneurysms and concurrently remove the tumor. After the surgery, we confirmed that both of the aneurysms were completely isolated, and the tumor was adequately resected. This patient had not suffered other neurological deficits. Unfortunately, her visual acuity had not recovered.

Conclusions: As we know, this is the first report of a unilateral surgical approach to clip the bilateral intracranial aneurysms and concurrently remove the tumor. It may provide a reference for clinical treatment of the similar disease.
\end{abstract}

Keywords: Bilateral aneurysms, Meningioma, Unilateral approach

\section{Background}

Comorbid brain tumor and aneurysm are rare and often identified accidentally [3, 8]. Pia et al. [7] reported a series of 23,876 brain tumor cases in 1972. The incidence of brain tumor and cranial aneurysm cooccurrence was approximately $0.2-0.7 \%$, and the mortality rate of the surgically treated patients was approximately $40 \%$ due to the complexity of the surgical site. With the development of medical equipment and microsurgical techniques, there has been a decreased mortality for this kind of diseases. The definite pathogenesis of the tumor and aneurysm is uncertain because

\footnotetext{
* Correspondence: chunhuizeng2015126@126.com

${ }^{\dagger}$ Equal contributors

'Department of Neurosurgery, The First Affiliated Hospital Nanchang

University, Yong wai zheng Street 17, Nanchang, Jiangxi Province 330006,

People's Republic of China

Full list of author information is available at the end of the article
}

of the rarity of this condition. Some literatures reported that the co-existence of aneurysms and tumors in the patient was possibly relevant with trauma, stereotactic radio surgery and genomics $[5,6]$, however, the relationship between aneurysms and tumors remains unclear. Here, we reported a case of a unilateral approach to the surgical treatment of comorbid tuberculum sellae meningioma and bilateral ICA aneurysms.

\section{Case presentation}

A 58-year-old female presented with a sudden onset of headache and nausea. The patient had a 5-year history of hypertension, she had no smoking history and family history. Three years earlier, she had been diagnosed with a sellar tumor associated with a right visual field deficit, but she did not receive medical treatment. One day after the onset of severe symptoms, she was examined to find 
a significant loss of light perception and a degenerated optic disc in the right eye with a further ophthalmological examination. A head computed tomography (CT) scan showed diffuse subarachnoid hemorrhage (SAH) in the cistern magna and carotid cistern with a roundshaped calcified mass in the sellar area (Fig. 1a). Magnetic resonance imaging (MRI) revealed that the lesion was slightly well-enhanced with gadolinium, which was the characteristic of meningioma (Fig. 1b, c). Consequently, a tuberculum sellae meningioma was diagnosed and measured at $2.5 * 3.0 * 2.2 \mathrm{~cm}$ in size. The MRI provided the evidence of damage to the right optic nerve canal and the anterior clinoid process. The relationship between the aneurysms and the tumor was clearly showed in preoperative CT angiography (CTA) and MRI (Fig. 1d, e). The 3-dimensional digital subtraction angiography (DSA) indicated that the tumor was coexisted with a right paraclinoid ICA aneurysm and a left ICA bifurcation aneurysm (Fig. 2). During the DSA, Balloon Occlusion Test (BOT) was performed, and the result was positive. Based on the head CT and DSA imaging findings, an unruptured, left side aneurysm was proposed. Neuroradiologists considered that the small left ICA bifurcation aneurysm was inappropriate for endovascular intervention. On the other hand, according to the head CT and MRI scan results, the ICA and aneurysm on the right side was not wrapped in the tumor, and just be pushed out and down.
The patient underwent a right pterion craniotomy. Twenty percent mannitol $250 \mathrm{ml}$ was intravenous dripped before we cut open the dura, so the intracranial pressure dropped and the right frontal lobe was retract. Then the sylvian fissure was completely split along the M1 and M2 segments of the middle cerebral artery. Part of the anterior clinoid process and platform of the sphenoid bone were grinded by drill, exposure plate of saddle tumor basal, the tuberculum sellae meningioma partly blocked the ICA and aneurysms, then, the tumor was decompressed with the help of CUSA (Cavitron Ultrasonic Surgical Aspirator; Sonoca300, Germany, Cicel), and removed piecemeal. Also the tumor grow into and damage the optic canal, the optic nerve, optic tract, and half of the chiasm on the right side has been reoriented from their original routes by the tumor, this part of tumor was exposed and removed by opening optic canal with a high-speed drill. The right ICA aneurysm was subsequently dissected and clipped after internal decompression of the meningioma (Fig. 3a, d). During the clipping, the right common carotid artery (CCA) and ICA was not exposed. We pressed the neck by hand for few minutes before the parent artery was blocked, and then the remaining tumor was removed, finally, according to the directionality of the left aneurysm, we dissected the opposite spaces in order to clip another aneurysm (Fig. 3b, e). After the surgery,
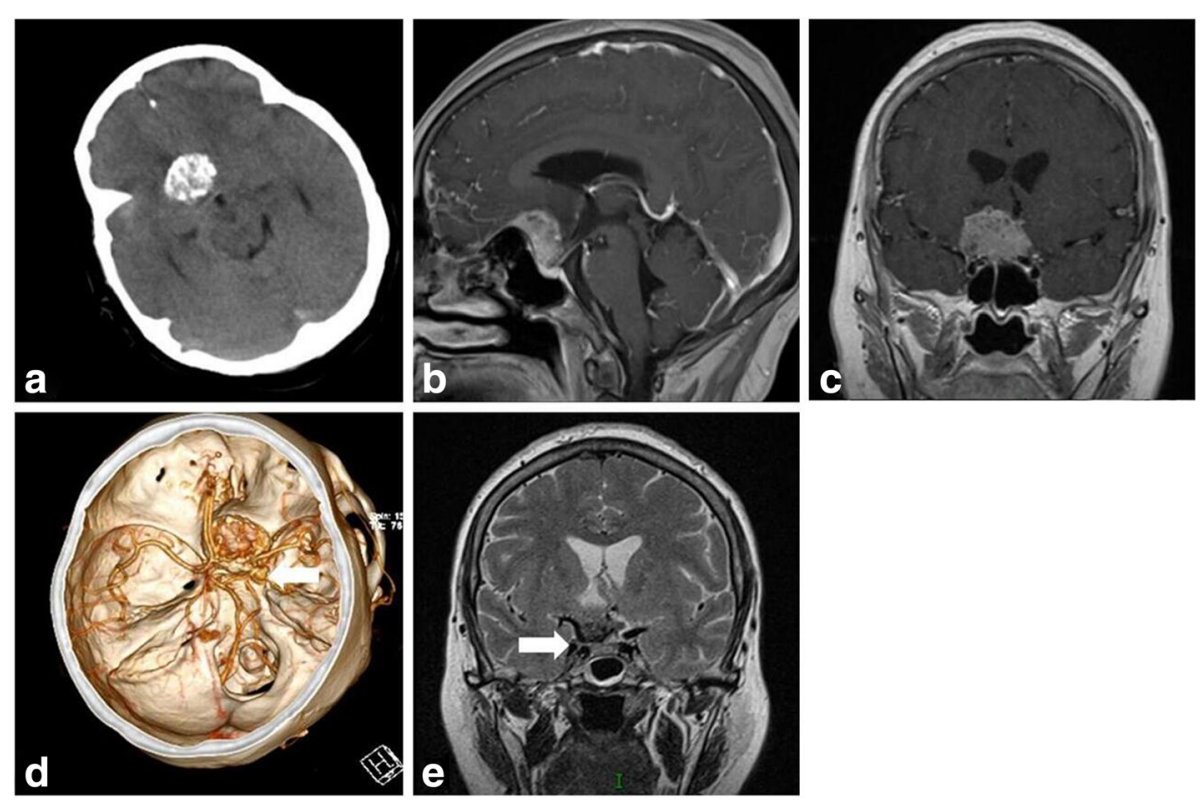

Fig. 1 Computed tomography (a) scan demonstrates a round-shaped calcified lesion close to the midline in the sellar region and a diffuse SAH in the cistern magna and carotid cistern. Preoperative magnetic resonance images contrast enhanced with gadolinium showing the sagittal (b) and coronal (c) views, which indicate a tuberculum sellae and in close contact with the bilateral ICA. $\mathbf{d}$ and $\mathbf{e}$ show that the right side aneurysm has been pushed out and down by the tumor. The arrows in (d) and (e) are both point to the right ICA aneurysm 

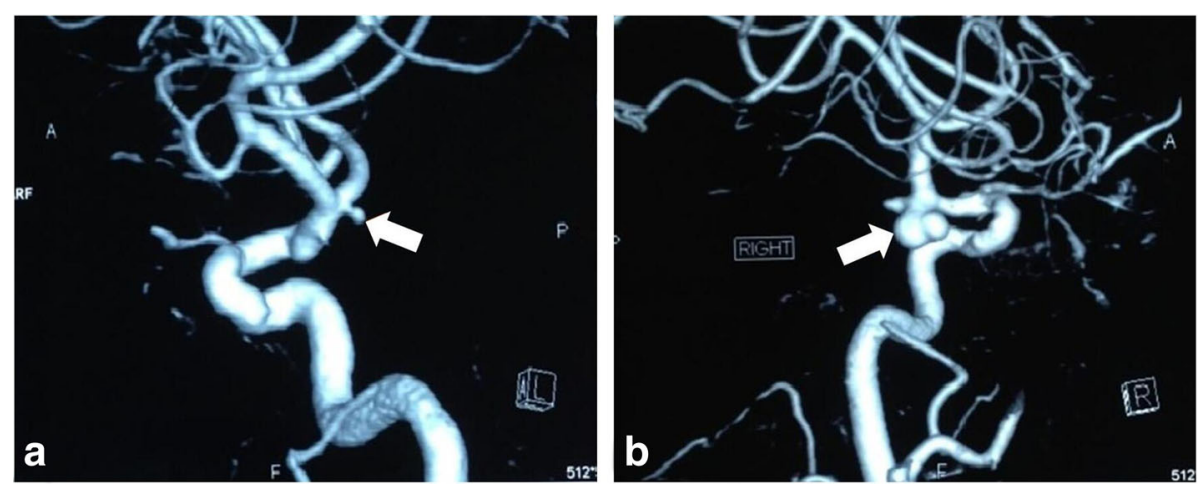

Fig. 2 Preoperative three-dimensional (3D) cerebral angiography, right (b) and left (a). Note the bilateral aneurysms of the ICA. The arrow in a and $\mathbf{b}$ separately point to the left and right ICA aneurysm

head CT angiography demonstrated that both of the aneurysms were completely isolated, and the tumor was adequately resected.

The post-operative course was uneventful. One week after the operation, the patient was subsequently discharged without new neurological deficits. Her visual acuity did not change after the operation. The histopathological diagnosis suggested that the tumor was a mixed meningioma (WHO Grade I). Follow-up imaging was conducted at 6 months post-surgery (Fig. 4); there was no residual tumor or tumor reoccurrence, whereas there was a fully occluded aneurysm.

\section{Discussion}

Most patients exhibited comorbid meningioma and pituitary adenomas rather than gliomas [8]. Fischer et al. [2] noted that coexisting lesions were always solitary in 95 consecutive patients with comorbid meningioma and aneurysm, whereas multiple occurrences were less frequent. Generally, the treatment strategy is aimed to resolve the main symptoms caused by the tumor or aneurysm; Yang et al. [9] reported a rare case which a ruptured paraophthalmic aneurysm was encased within meningioma and a contralateral extradural aneurysm. They thought treatment strategy for comorbid disease
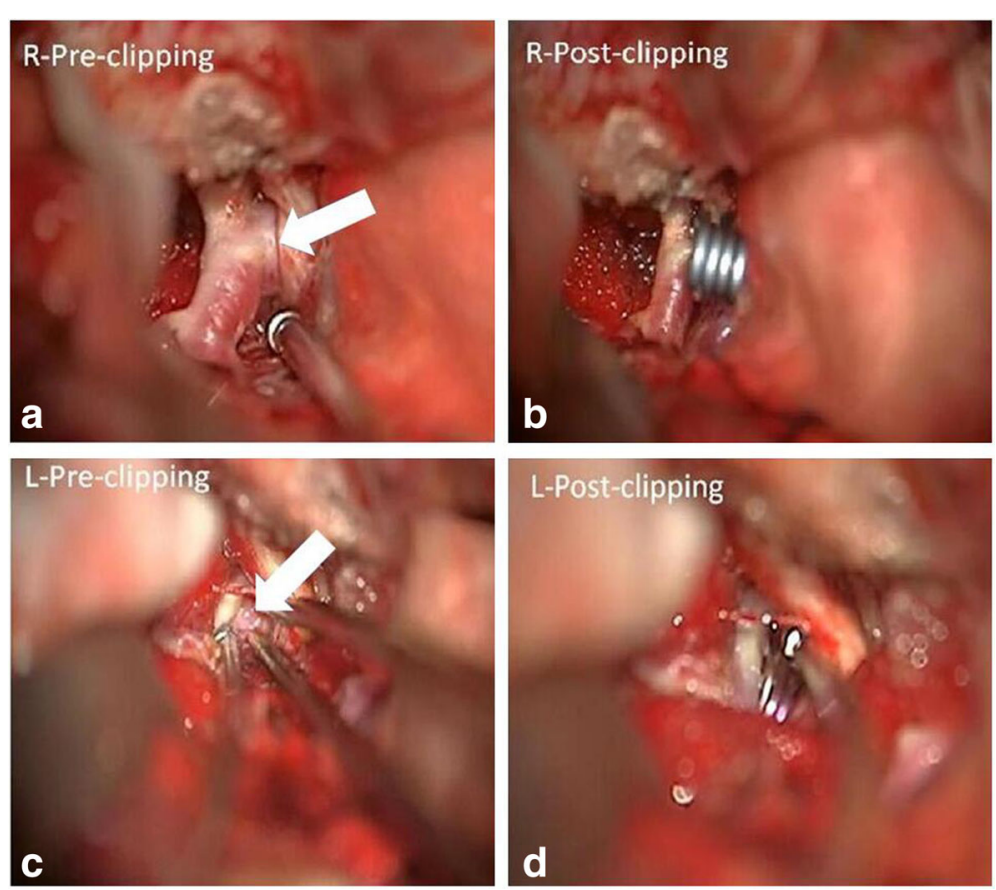

Fig. 3 Intraoperative view after a right pterional craniotomy, right pre-clipping (a), right post-clipping (b), left pre-clipping (c) and left post-clipping (d). Note the bilateral ICA aneurysms. Clip reconstruction after completing tumor resection 


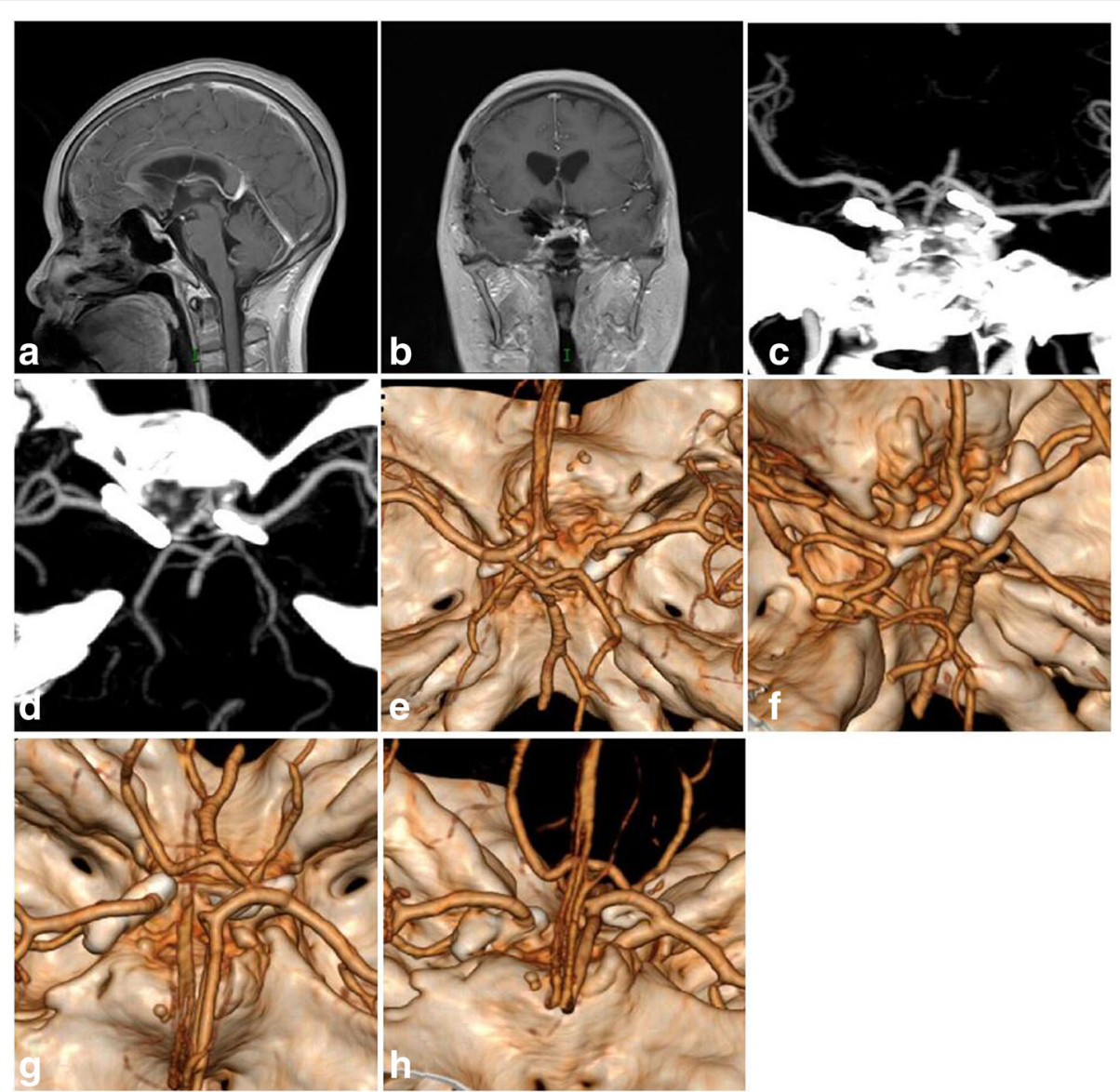

Fig. 4 The post-operative MRI of surgery showed the sagittal (a) and coronal (b) views, and both of the ICA aneurysms were well clipped showed in (c-h)

that handled both of the aneurysm and the tumor was recommended. However, there have been controversies regarding bilateral or multiple lesions. Bilateral craniotomies are frequently applied to clip the bilateral or multiple aneurysms, including an initial craniotomy for the ruptured aneurysm, and a second craniotomy for the un-ruptured contralateral aneurysm in one session or multiple sessions. Considering the locations of both aneurysms and new microsurgical techniques, De Sousa et al. [1] has reported that several cases with bilateral multiple intracranial aneurysms were clipped through a unilateral approach, even the "mirror-image" middle cerebral artery was involved. We have previously reported a series of 12 patients with bilateral multiple intracranial aneurysms that were treated unilaterally [4]. However, as we know, this report describes the first case of comorbid bilateral intracranial aneurysms and tuberculum sellae meningioma treated with a unilateral surgical approach, so it may have not too much to compare with other reports. For this case, the benefits and risks of using this method to treat comorbid bilateral aneurysms and meningioma were mainly evaluated below.
The right side of surgical approach was chose according to the ruptured aneurysm. The head CT scan showed that the blood of the subarachnoid space in the right sylnian cistern carotid cistern and supra sella cistern was more than the left side, the right ICA aneurysm is bigger than the left one, and it has a daughter bubbles. So the right ICA aneurysm was considered as the ruptured one. The advantages of this surgery is through single side surgical approach to reduce the trauma and complications rather than bilateral surgical approach; If we applied endovascular treatment, the patient had to take anticoagulant drugs which may led to a lot of bleeding and add more difficulty of the tumor hemostasis when we removed the tumor; The risks of this kind of surgical approach is the re-rupture of aneurysms, it usually happens when the aneurysm is wrapped in the tumor, however, according to the head CT and MRI scan results, the ruptured right ICA aneurysm was only pushed aside by the tumor, rather than be wrapped in the tumor. On the other hand, during the surgery, we grinded off part of the anterior clinoid process to expose the proximal end of intracranial ICA to control the bleeding and 
rupture of aneurysm. The coexistence of multiple aneurysms and tumors exhibits a rare prevalence and high mortality, which implies the need for additional recognition regarding this clinical condition. The unilateral surgical approach for bilateral aneurysms is limited to the aneurysm location, brain edema, and temporary occlusion of the parent artery. Multiple aneurysms may be simultaneously occluded with an endovascular approach (e.g., coil embolization or balloon occlusion). If aneurysms are adjacent or encased in a tumor, endovascular treatment may firstly be performed to reduce the bleeding risk following tumor resection; however, if we performed endovascular treatment for the right one first, and then resect the tumor from left craniotomy and clip the left aneurysm as well, the stent may probably be used in the endovascular treatment for the right aneurysm. So, the patient have to take antiplatelet medication (such as aspirin or clopidogrel) after the surgery which may add the bleeding risk during we cut out the tumor. On the other hand, the base of tumor was mainly located on the right side, the resection of this tumor from left side will suffer poor surgical field. For this patient, it also could be embolized with conventional coiling, without stent-assisted technique, however, the patient had to suffer two surgeries. If the patient has sufficient funds and agrees to undertake common risks voluntarily, the way of two-time operation may also be applied. We provided several choices of surgical approachs to this patient. Finally, they decided to do the one staged surgery. With the improvement of surgical conditions, sufficient evaluation before surgery and excellent control of parent arteries, from the good outcome of this patient as well. Mutiple intracranial lesions treated by one surgical approach in one staged operation may be a good choice for part of patients.

\section{Conclusions}

This novel and rare case reported an efficacious unilateral surgical approach for the treatment of comorbid bilateral intracranial aneurysms and tuberculum sellae meningioma, which may provide a reference for other neurosurgical teams when they face with similar cases.

\author{
Acknowledgements \\ Not applicable. \\ Funding \\ None. \\ Availability of data and materials \\ Please contact author for data requests.
}

\section{Authors' contributions}

JF and MW acquired the data, analyzed the data and wrote the manuscript. CZ conceived and designed the manuscript. TH interpreted the data. RF All of the authors critically revised the manuscript, read and approved the final manuscript

Competing interests

The authors declare that they have no competing of interests.
Consent for publication

Consent for publication of this case has been acquired from the patient involved.

Ethics approval and consent to participate

Not applicable.

\section{Author details}

'Department of Neurosurgery, The First Affiliated Hospital Nanchang University, Yong wai zheng Street 17, Nanchang, Jiangxi Province 330006, People's Republic of China. ${ }^{2}$ Department of Functional Diagnosis, The Affiliated Hospital of Jiangxi University of Traditional Chinese Medicine, Nanchang, Jiangxi Province, China.

Received: 4 March 2016 Accepted: 14 January 2017

Published online: 01 May 2017

\section{References}

1. de Sousa AA, Filho MA, Faglioni Jr W, Carvalho GT. Unilateral pterional approach to bilateral aneurysms of the middle cerebral artery. Surg Neurol. 2005;63 Suppl 1:1-7.

2. Fischer BR, Palkovic $S$, Holling $M$, Niederstadt $T$, Jeibmann $A$, Wassmann $H$. Coexistence of cerebral aneurysm and meningioma-pure accident? Clin Neurol Neurosurg. 2009;111:647-54.

3. Handa J, Matsuda I, Handa H. Association of brain tumor and intracranial aneurysms. Surg Neurol. 1976;6:25-9.

4. Hong T, Wang Y. Unilateral approach to clip bilateral multiple intracranial aneurysms. Surg Neurol. 2009;72 Suppl 1:S23-8. discussion S8.

5. Katsumi H, Yuhei Y, Masahiro S, Shigeru N. Rupture of an internal carotid artery aneurysm within a clinoidal meningioma following stereotactic radiosurgery. Acta Neurochir. 2011;153(10):1995-6.

6. O'Neill OR, Barnwell SL, Silver DJ. Middle meningeal artery aneurysm associated with meningioma: case report. Neurosurgery. 1995;36(2):396-8.

7. Pia HW, Obrador S, Martin JG. Association of brain tumors and arterial intracranial aneurysms. Acta Neurochir. 1972;27:189-204.

8. Spitler K, Drazin D, Hanna G, Patel A, Chu R. Association of intracranial aneurysms with meningiomas, pituitary adenomas, and gliomas: review of possible interrelationships. Isrn Neurology. 2013;2013:383425.

9. Yang W, Huang J. Meningioma encased ruptured paraophthalmic aneurysm: Case report and review of the literature. Interdiscip Neurosurg. 2014;1(2):17-20
Submit your next manuscript to BioMed Central and we will help you at every step:

- We accept pre-submission inquiries

- Our selector tool helps you to find the most relevant journal

- We provide round the clock customer support

- Convenient online submission

- Thorough peer review

- Inclusion in PubMed and all major indexing services

- Maximum visibility for your research

Submit your manuscript at www.biomedcentral.com/submit
Ciomed Central 